\title{
Efficacy of Hypertonic Saline-Epinephrine Local Injection Around the Anal Side before Endoscopic Papillectomy for Ampullary Tumors
}

\author{
Naoki Okano, Yoshinori Igarashi, Ken Ito, Saori Mizutani, Hiroki Nakagawa, Kouji Watanabe, Yuuto Yamada, Kensuke Yoshimoto, \\ Yuusuke Kimura, Susumu Iwasaki, Kensuke Takuma, Seiichi Hara and Yuui Kishimoto \\ Division of Gastroenterology and Hepatology, Toho University Omori Medical Center, Tokyo, Japan
}

Background/Aims: Bleeding is a complication of endoscopic snare papillectomy for ampullary tumors. This study aimed to investigate the clinical efficacy of hypertonic saline-epinephrine (HSE) local injection before endoscopic papillectomy for prevention of bleeding.

Methods: We retrospectively reviewed the data of 107 consecutive patients with ampullary tumors who underwent endoscopic papillectomy. The rates of en bloc resection, pathological resection margins, and prevention of immediate or delayed bleeding in the simple snaring resection group (Group A) and the HSE injection group (Group B) were compared.

Results: A total of 44 and 63 patients were enrolled in Groups A and B, respectively. The total complete resection rate was $89.7 \%$ (96/107); the clinical complete resection rates in Group A and Group B were 86.3\% (38/44) and 92.1\% (58/63), respectively ( $p=0.354)$. Post-papillectomy bleeding occurred in 22 patients. In Groups A and B, the immediate bleeding rates were 20.5\% $(9 / 44)$ and $4.8 \%$ (3/63), respectively $(p=0.0255)$, while the delayed bleeding rates were $7 \%(3 / 44)$ and $11 \%(7 / 63)$, respectively $(p=0.52)$. The rates of positive horizontal and vertical pathological margin in both groups were $27 \%$ and $16 \%$, respectively.

Conclusions: HSE local injection was effective in preventing immediate bleeding and was useful for safely performing endoscopic papillectomy for ampullary tumors. Clin Endosc 2021;54:706-712

Key Words: Ampullary tumor; Complication; Endoscopic papillectomy; Local injection

\section{INTRODUCTION}

In recent years, advances in endoscopic diagnosis and treatment of pancreaticobiliary diseases have led to the endoscopic treatment of duodenal ampullary tumors. ${ }^{1-7}$ Most duodenal ampullary tumors are derived from adenomas or carcinomas; however, adenomas are considered precancerous lesions that

Received: August 24, 2020 Revised: November 11, 2020

Accepted: December 2, 2020

Correspondence: Naoki Okano

Division of Gastroenterology and Hepatology, Toho University Omori Medical Center, 6-11-1, Oomorinishi, Oota-ku Tokyo 143-8541, Japan

Tel: +81-3-3762-4151, Fax: +81-3-3763-8542, E-mail: n-okano@med.toho-u.ac.jp ORCID: https://orcid.org/0000-0002-9759-1374

(c) This is an Open Access article distributed under the terms of the Creative Commons Attribution Non-Commercial License (http://creativecommons.org/ licenses/by-nc/3.0) which permits unrestricted non-commercial use, distribution, and reproduction in any medium, provided the original work is properly cited. may become carcinomas through an adenoma-carcinoma sequence. ${ }^{89}$ Thus, complete resection is essential for radical treatment. Traditionally, local papillectomy and pancreaticoduodenectomy have been the standard treatments for duodenal ampullary tumors. However, the invasiveness of these procedures poses a great challenge among older patients and those with comorbidities. Therefore, surgical treatment was replaced with endoscopic papillectomy, which is a minimally invasive treatment, but the excision procedure has not been standardized.

The duodenal papilla is a rounded projection at the orifice of the pancreaticobiliary duct; the complications associated with surgical excision of this part include pancreatitis and cholangitis. Furthermore, treatment difficulties may arise when bleeding or perforation occurs due to the limitations in the devices used during duodenoscopy.

Submucosal injections of saline or diluted epinephrine 
solution have been administered to achieve a safe and complete resection of tumors and to prevent the occurrence of complications, such as bleeding and perforation from endoscopic mucosal resection (EMR) of the stomach and colorectal adenomas. A local injection of hypertonic saline-epinephrine (HSE) is also considered useful in endoscopic resection of early gastric cancer. ${ }^{10}$ Since 2010 , HSE local injection has been performed in our institution for the prevention of bleeding. However, a preoperative submucosal injection is generally not performed in patients scheduled for endoscopic papillectomy; the tumor may not appear elevated because the duodenal papilla is located at the orifice of the pancreaticobiliary duct.

The utility of preoperative submucosal injections for improving the complete resection rate or preventing complications, such as bleeding, pancreatitis, and perforation, during endoscopic papillectomy needs to be clarified further. Therefore, this study aimed to investigate the clinical efficacy of HSE local injection around the anal side before endoscopic papillectomy for the prevention of bleeding.

\section{MATERIALS AND METHODS}

Data of consecutive patients who underwent endoscopic papillectomy for duodenal ampullary tumors in our institution between October 2002 and September 2019 were retrospectively reviewed. The complete resection rates, pathological margins, and post-papillectomy complications were compared between patients who underwent simple snaring resection (Group A) before 2010 and those who received a pre-procedure submucosal HSE injection (Group B) after 2010.

Endoscopic papillectomy was performed by two endoscopists with more than 15 years of experience performing endoscopic retrograde cholangiopancreatography (ERCP). Computed tomography, endoscopic ultrasound, and ERCP were performed preoperatively, and endoscopic papillectomy was indicated in patients with (1) exposed tumor type, (2) no tumor extension to the biliary and pancreatic ducts, and (3) no tumor extension to the duodenal muscle layer. Endoscopic papillectomy was not indicated in patients with ulceration because of their strong potential for deep invasion. In cases where preoperative biopsy revealed adenocarcinoma, endoscopic papillectomy was performed in patients who satisfied the abovementioned criteria; in patients without lymph node metastasis, endoscopic papillectomy was performed after obtaining an informed consent. This study was approved by the ethics committee of our institution (M16155). Informed consent was obtained from all patients.

\section{Procedure}

Endoscopic papillectomy was performed under a duodenoscope (TJF-240 or TJF-260V; Olympus, Tokyo, Japan) with a working channel of $4.2 \mathrm{~mm}$. After an indigo carmine dye was sprayed to confirm the extent of the lesion, en bloc excision was attempted using a single-line snare or SD-230U (Olympus, Tokyo, Japan) to snare from the oral side. In Group B, a small amount (1-2 mL) of HSE, consisting of saline (15 mL), 10\% sodium chloride solution $(5 \mathrm{~mL})$, and epinephrine $(1 \mathrm{~mL})$, was injected into the submucosal layer around the anal side after spraying an indigo carmine dye. Excision was performed with a high-frequency electrosurgical generator in Autocut $120 \mathrm{~W}$ mode using ICC200 (Erbe, Tuebingen, Germany) or PSD-60 (Olympus, Tokyo, Japan). Snaring was initiated at the oral side of the papilla and was advanced further to fit the entire tumor; then, the anal papilla was resected (Fig. 1). After collecting the specimen with a net catheter, the excision site was assessed. If residual tumor was suspected, additional snare resection was performed. For bleeding, hemostasis was achieved through high-frequency coagulation using a snare tip, argon plasma coagulation, or application of hemoclips. Pancreatic stenting or nasal pancreatic duct drainage was performed to prevent postoperative pancreatitis, except in the patients with pancreatic divisum. Biliary stenting was performed at the operator's discretion. Bleeding that occurred during the endoscopic papillectomy procedure was defined as immediate bleeding, while bleeding that occurred $12 \mathrm{hr}$ after the endoscopic papillectomy procedure was defined as delayed bleeding. Blood tests were performed $2 \mathrm{hr}$, the next morning, 3 day, and 7 day after papillectomy to assess for complications. If anemia, hematemesis, or melena progression was observed, endoscopic hemostasis was attempted. Postoperative pancreatitis was evaluated using Cotton's criteria. Follow-up examinations via a duodenoscope were conducted 3 or 6 months after resection and yearly for 1-5 years. If recurrence was suspected, a biopsy was performed for pathological evaluation. Complete clinical resection was defined as the absence of recurrence 3-6 months after resection.

\section{Statistical analysis}

All statistical analyses were performed using EZR (Saitama Medical Center, Jichi Medical University, Saitama, Japan), which is a graphical user interface for $\mathrm{R}$ (The R Foundation for Statistical Computing, Vienna, Austria). ${ }^{11}$ Continuous and categorical variables were compared using the Mann-Whitney $U$ test and $\chi^{2}$ test. Two-sided $p$-values of $<0.05$ were considered significant. 

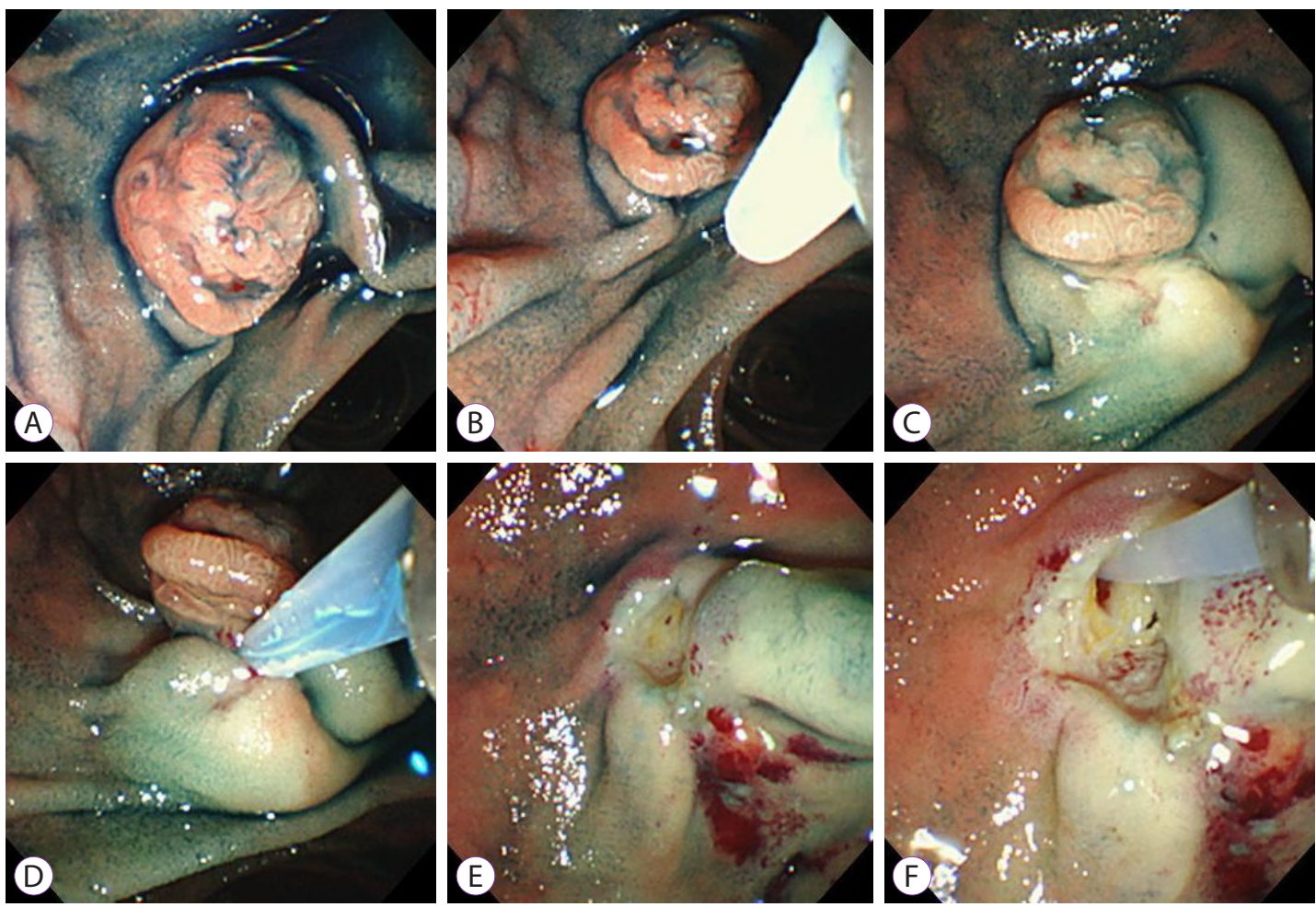

Fig. 1. Hypertonic saline-epinephrine (HSE) submucosal injection before papillectomy. Endoscopic view of ampullary adenoma. (A) Endoscopic view of ampullary adenoma. (B) HSE submucosal injection around the anal side of the duodenal papilla. (C) The anal side of the papilla appeared white after HSE injection. (D) Snaring with safety margin. (E) No bleeding after papillectomy. (F) The orifice of the bile duct was clearly identified.

\section{RESULTS}

A total of 113 patients underwent endoscopic papillectomy at our institution between October 2002 and September 2019. Six patients with histologically non-neoplastic lesions were excluded. Finally, 107 patients were enrolled in this study: 44 in Group A (22 men: average age, $66.43 \pm 12.97$ years) and 63 in Group B (30 men: average age, $67 \pm 11$ years). The final pathological findings of Group A were as follows: 24 had adenomas, 8 had adenoma-like adenocarcinoma, 11 had adenocarcinomas, and 1 had neuroendocrine tumor (NET). Meanwhile, the final pathological findings of Group B were as follows: 54 had adenomas, 3 had adenoma-like adenocarcinoma, 5 had adenocarcinomas, and 1 had NET. The mean tumor sizes of Groups A and B were $16.93 \pm 7.29 \mathrm{~mm}$ and $17.59 \pm 7.56 \mathrm{~mm}$, respectively. The total en bloc resection rate was $89.7 \%(96 / 107)$. The en bloc resection rates in Groups S and I were $86.3 \%$ (38/44) and $92.1 \%$ (58/63), respectively; although the en bloc resection rate in Group B tended to be higher, the difference was not statistically significant $(p=0.354)$. Complete clinical resection was achieved in $89.7 \%$ of the patients (102/107): Group A: $88.6 \%$ (39/44) and Group B: 90.5\% (57/63) $(p=0.754)$ (Table 1).

In Group $\mathrm{A}$, the pathological examination revealed that $64 \%(28 / 44)$ had negative, $27 \%(12 / 44)$ had positive, and $9 \%$ $(4 / 44)$ had uncertain vertical resection margin; in Group B, $67 \%(42 / 63)$ had negative, $16 \%$ (10/63) had positive, and $17 \%$ $(11 / 63)$ had uncertain vertical resection margin. In Group A, $64 \%$ (28/44) had negative, 27\% (12/44) had positive, and 9\% (4/44) had uncertain horizontal resection margin; in Group B, $71 \%$ (45/63) had negative, $16 \%$ (10/63) had positive, and $13 \%$ (8/63) had uncertain horizontal resection margin. It was difficult to evaluate the resection margin in 11 patients who had a piecemeal resection; in the other patients, it was difficult to determine the pathological resection margin due to the burning effect of endoscopic papillectomy. The positive rates of horizontal and vertical pathological margins were lower in Group B, but no statistically significant difference was observed (Table 2).

Post-papillectomy bleeding occurred in 22 (20.6\%) patients: 12 (27.2\%) in Group A and 10 (15.9\%) in Group B. Immediate bleeding occurred in 20.5\% (9/44) of the patients in Group 
Table 1. Clinicopathological Characteristics of Patients with Ampullary Tumor

\begin{tabular}{|c|c|c|c|}
\hline & $\begin{array}{c}\text { A group } \\
(n=44)\end{array}$ & $\begin{array}{l}\text { B group } \\
(n=63)\end{array}$ & $p$-value \\
\hline Gender (M:F) & $22: 22$ & $30: 33$ & 0.85 \\
\hline Mean age, yr & $66.43 \pm 12.97$ & $67.0 \pm 11$ & 0.66 \\
\hline Average tumor size, $\mathrm{mm}$ & $16.93 \pm 7.29$ & $17.59 \pm 7.56$ & 0.66 \\
\hline \multicolumn{4}{|l|}{$\begin{array}{l}\text { Final histopathological } \\
\text { result }\end{array}$} \\
\hline Adenoma & 24 & 54 & \\
\hline Carcinoma in adenoma & 8 & 3 & \\
\hline Carcinoma & 11 & 5 & \\
\hline NET & 1 & 1 & \\
\hline En bloc resection & $38(86.3 \%)$ & $58(92.1 \%)$ & 0.35 \\
\hline Clinical complete resection & $39(88.6 \%)$ & $57(90.5 \%)$ & 0.75 \\
\hline Pacreatic stent placement & $33(75 \%)$ & $50(79 \%)$ & 0.323 \\
\hline \multicolumn{4}{|l|}{ Adverse events } \\
\hline \multicolumn{4}{|l|}{ Bleeding } \\
\hline Immediate & $9(20.5 \%)$ & $3(4.8 \%)$ & 0.026 \\
\hline Delayed & $3(6.8 \%)$ & $7(11.1 \%)$ & 0.52 \\
\hline Pacreatitis & $14(31.8 \%)$ & $7(11.1 \%)$ & 0.010 \\
\hline Perforation & 0 & $1(1.6 \%)$ & 1 \\
\hline Mortality & 0 & 0 & \\
\hline
\end{tabular}

NET, neuroendocrine tumor.

Table 2. Results of Pathological Margin

\begin{tabular}{lccc}
\hline & $\begin{array}{c}\text { A group } \\
(\boldsymbol{n}=\mathbf{4 4})\end{array}$ & $\begin{array}{c}\text { B group } \\
(\boldsymbol{n}=\mathbf{6 3})\end{array}$ & $\boldsymbol{p}$-value \\
\hline Vertical margin & & & \\
Positive & $12(27 \%)$ & $10(16 \%)$ & 0.22 \\
Negative & $28(64 \%)$ & $42(67 \%)$ & \\
Uncertain & $4(9 \%)$ & $11(17 \%)$ & \\
Horizontal margin & & & \\
Positive & $12(27 \%)$ & $10(16 \%)$ & 0.22 \\
Negative & $28(64 \%)$ & $45(71 \%)$ & \\
Uncertain & $4(9 \%)$ & $8(13 \%)$ & \\
\hline
\end{tabular}

Table 3. Immediate Bleeding Cases Compaired wtih Tumor Size

\begin{tabular}{lcc}
\hline Tumor size & Immediate bleeding & $\boldsymbol{p}$-value \\
\hline$\leqq 10 \mathrm{~mm}$ & $0 \%(0 / 20)$ & 0.004 \\
$11-19 \mathrm{~mm}$ & $6.4 \%(3 / 47)$ & \\
$20 \mathrm{~mm} \leqq$ & $25 \%(10 / 40)$ & \\
\hline
\end{tabular}

A and in $4.8 \%(3 / 63)$ of the patients in Group B $(p=0.0255)$. Delayed bleeding occurred in $6.8 \%(3 / 44)$ of the patients in Group A and 11.1\% (7/63) of those in Group B ( $p=0.52)$. One patient in Group B, who had taken docosahexaenoic acid and eicosapentaenoic acid, experienced delayed bleeding that required transfusion and arterial embolization.

Post-papillectomy pancreatitis occurred in 21 (19.6\%) patients: 14 (31.8\%) in Group A and 7 (11.1\%) in Group B $(p=0.0124)$. In addition, immediate bleeding and pancreatitis occurred simultaneously in $11.4 \%(5 / 44)$ and $1.6 \%(1 / 63)$ of the patients in Group A and Group B, respectively $(p=0.0794)$. Perforation occurred in one patient in Group B, who recovered conservatively. No procedure-related mortalities were reported (Table 1).

Among the cases of immediate bleeding, the incidence of bleeding was associated with tumor size: larger tumors had a tendency to cause bleeding (Table 3). Furthermore, we evaluated the subgroups according to the pathological findings. Immediate bleeding occurred in $10.3 \%$ (8/78) of the patients in the adenoma subgroup and in $14.8 \%(4 / 27)$ of the patients in the carcinoma and CiA subgroup, relatively. There were no statistical differences between the pathological findings (Table 4). The logistic regression model was used to calculate the odds ratios (ORs) and their corresponding 95\% confidence intervals (CIs) for immediate bleeding associated with tumor size, HSE submucosal injection, and carcinoma. Results showed an OR of 9.61 for tumor size (95\% CI, 2.255-40.96; $p=0.002)$, an OR of 0.173 for HSE submucosal injection ( $95 \%$ CI, $0.044-0.679 ; p=0.012$ ), and an OR of 1.04 for carcinoma (95\% CI, 0.208-5.200; $p=0.963$ ) (Table 5).

Table 4. Immediate Bleeding Cases Compaired wtih Pathological Findings

\begin{tabular}{lcc}
\hline Pathological findings & Immediate bleeding & $\boldsymbol{p}$-value \\
\hline Adenoma & $10.3 \%(8 / 78)$ & 0.526 \\
CiA/Carcinoma & $14.8 \%(4 / 27)$ & \\
\hline
\end{tabular}

CiA, carcinoma in adenoma.

Table 5. Odds Ratios for Immediate Bleeding Cases

\begin{tabular}{lccc}
\hline Characteristics & Odds ratios & $\mathbf{9 5 \%}$ CI & p-value \\
\hline Carcinoma & 1.040 & $0.208-5.200$ & 0.963 \\
HSE local injection & 0.173 & $0.044-0.679$ & 0.012 \\
Tumor size $(>20 \mathrm{~mm})$ & 9.61 & $2.255-40.96$ & 0.002 \\
\hline
\end{tabular}

$\mathrm{CI}$, confidence interval; HSE, hypertonic saline-epinephrine. 


\section{DISCUSSION}

Endoscopic papillectomy has been performed in recent years, as a minimally invasive treatment for adenomas. ${ }^{12}$ Although the endoscopic papillectomy procedure is not standardized, it has a high complete resection rate. Submucosal injection of saline or a dilute solution of epinephrine is commonly administered to achieve a safe and a complete resection and to prevent the occurrence of complications associated with EMR for stomach and colorectal tumors. ${ }^{11}$ In endoscopic papillectomy, submucosal local injection before resection is generally not performed because (1) the tumor cannot be lifted since the duodenal papilla is located at the orifice of the pancreaticobiliary duct; (2) the tumor is depressed by local injection of the abovementioned solution, making snaring and en bloc resection difficult; and (3) disadvantages, such as increase in the risk of postoperative pancreatitis, were noted. ${ }^{13}$ On the contrary, submucosal injection may prevent unnecessary resections because the inability to lift the lesion is predictive of invasion; thus, the risk of bleeding can be reduced. ${ }^{14}$ Moreover, submucosal injection was useful for achieving safe excision of lateral spreading tumors. ${ }^{15}$

The complete resection rate of endoscopic papillectomy was $46 \%-92 \% .{ }^{12}$ Hyun et al. compared the complete resection rate between the submucosal injection group with epinephrine solution (1:10,000 dilution) and the no-injection group and found that the resection rate in the submucosal injection group was significantly lower (50\% vs. $80.8 \%) .{ }^{16}$ Local injection caused the ampullary tumor to sunk and made the tumor margin unrecognizeable. ${ }^{16}$ In our method, which involved the administration of HSE submucosal injection around the anal side, the submucosal injection group had a slightly higher clinical complete resection rate ( $90.5 \%$ vs. $88.6 \%$ ).

A few studies have reported the pathological margins of endoscopic papillectomy. Hyun et al. reported the positive rates of horizontal and vertical margins in a submucosal injection group and a no injection group $(8.3 \%$ vs. $7.7 \%$ and $20.8 \%$ vs $7.7 \%){ }^{16}$ Although no statistical difference was found, the positive rates of the horizontal and vertical margins between the submucosal injection group and the no-injection group were both $16 \%$ and $27 \%$ in our study. The difference in injection method was considered to be the reason why the positive rate of the pathological margins tended to be lower in the submucosal injection group. In previous studies, many institutions performed a local injection around the entire papilla, and the amount of injection varied. ${ }^{17}$ In our institution, local injection was not performed around the oral side; rather, only a small amount (1-2 mL) of solution was injected around the anal side. If local injection is performed around the entire papilla, the ampullary tumor may be depressed by local injection, but the possibility decreases if only a small amount of solution is locally injected around the anal side. Furthermore, if snaring is initiated from the oral side, the anal margin of the tumor may be difficult to identify. By injecting a small amount around the anal side, the tumor margin can be easily identified, and more reliable snaring is possible.

The incidence rates of bleeding and pancreatitis were reported to be $2 \%-30 \%$ and $3 \%-25 \%$, respectively. ${ }^{18}$ Generally, pancreatic stenting is performed to prevent postoperative pancreatitis. ${ }^{18-20}$ However, only a few studies have reported the prevention of bleeding. Hemostasis of intraoperative bleeding may take time, which may increase the risk of pancreatitis. In our study, intraoperative hemorrhage and pancreatitis tended to occur more frequently in the group without local injection. Previous studies reported that since endoscopic hemostasis was unsuccessful, surgical hemostasis was performed; moreover, pancreatic duct stent occlusion due to a blood clot resulted in the occurrence of pancreatitis. ${ }^{5,21}$ Saline and diluted epinephrine solution have been used for submucosal injection in previous studies, ${ }^{14,16,17}$ but their usefulness remained controversial. Hyun et al. compared a group receiving submucosal injection of diluted epinephrine solution before resection $(n=24)$ with a group not receiving an injection $(n=26) .^{16}$ They reported that there was no difference in the percentage of bleeding. ${ }^{16}$ On the contrary, Desilets et al. suggested the usefulness of diluted epinephrine solution $(3-10 \mathrm{~mL})$ injections for the prevention of bleeding in 41 patients. ${ }^{14}$ In the present study, we administered a submucosal injection using HSE before resection, and the percentage of immediate bleeding was significantly less in the local injection group. HSE has a vasospastic effect; hypertonic saline prolongs the pharmacological effect of epinephrine and causes tissue swelling; it causes hemostasis due to the formation of thrombus in blood vessels. Therefore, the standard dilution of epinephrine solution must be used for the prevention of bleeding.

There is no consensus on the optimal power output and mode of electrosurgical current for endoscopic papillectomy. Power output ranged from 30 to $150 \mathrm{~W}^{3,4,6,14,22}$ Both pure-cutting current and blended current have been used, and neither has been proven to be superior to the other. Some investigators advocate that the use of pure-cutting mode increases the risk of bleeding. By contrast, blended current may potentially cause tissue edema due to the coagulation effect. If HSE injection reduces bleeding, it can be used as an optimal method.

Although local injection has been reported to increase the risk of pancreatitis after endoscopic papillectomy, ${ }^{13}$ our study showed fewer cases of pancreatitis. Furthermore, pancreatic stent placement was more common in the injection group, 

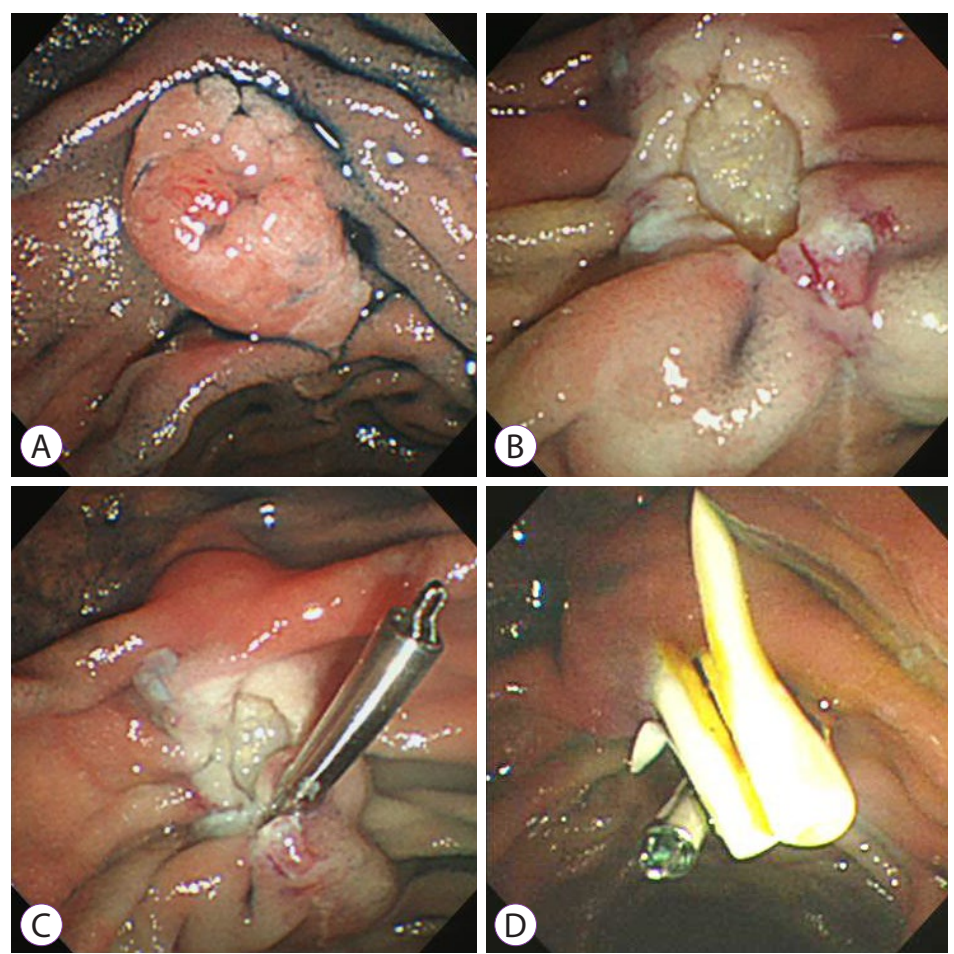

Fig. 2. Reefing with hemoclips after papillectomy. (A) Endoscopic view of ampullary adenoma. (B) After papillectomy with hypertonic saline-epinephrine injection. (C) Reefing with hemoclips to the frenulum side of the duodenal papilla. (D) Biliary and pancreatic stents were placed.

while postoperative pancreatitis tended to be lower in this injection group. Frequently, bleeding and pancreatitis occurred simultaneously in our study, indicating that the time spent in achieving hemostasis may have affected the prompt identification of the pancreatic duct. Local injection before resection is considered useful for securing a sufficient endoscopic view. However, in our study, delayed bleeding tended to occur more frequently in the HSE local injection group, and countermeasures were required. The pharmacological effect of HSE is time dependent, and it is important to prevent delayed bleeding using an additional method. Moreover, hemoclips are applied to prevent postoperative bleeding. Clipping, in addition to HSE submucosal injection, is expected to facilitate the prevention of immediate and delayed bleeding after endoscopic papillectomy (Fig. 2).

The current study has several limitations. First, it was a single-center and retrospective study; therefore, the number of patients used in the study was small. Second, the learning curve might have affected the incidence of complications. In the future, larger scale randomized controlled trials are required to confirm our results.

In conclusion, our findings demonstrate that a submucosal
HSE local injection around the anal side prior to resection is safe and facilitates the prevention of intraoperative bleeding. Moreover, the application of hemoclips, in addition to the submucosal injection method, is expected to further decrease the complication rate.

Conflicts of Interest

The authors have no potential conflicts of interest.

Funding

None.

Author Contributions

Conceptualization: Naoki Okano, Yoshinori Igarashi, Ken Ito

Data curation: NO, Saori Mizutani, Hiroki Nakagawa, Kouji Watanabe, Yuuto Yamada, Kensuke Yoshimoto, Yuusuke Kimura, Susumu Iwasaki, Kensuke Takuma, Seiichi Hara and Yuui Kishimoto

Formal analysis: NO, SM, HN, KW, YY, KY, YK, SI, KT, SH, YK

Writing-original draft: $\mathrm{NO}$

Writing-review\&editing: NO, YI

ORCID

Naoki Okano:

Yoshinori Igarashi:

Ken Ito: https://orcid.org/0000-0002-9759-1374

https://orcid.org/0000-0002-9345-1649 https://orcid.org/0000-0001-5559-0990 
Saori Mizutani:

Hiroki Nakagawa:

Kouji Watanabe:

Yuuto Yamada:

Kensuke Yoshimoto:

Yuusuke Kimura:

Susumu Iwasaki:

Kensuke Takuma:

Seiichi Hara:

Yuui Kishimoto: https://orcid.org/0000-0001-6242-7673 https://orcid.org/0000-0001-9077-9289 https://orcid.org/0000-0001-7636-4001 https://orcid.org/0000-0002-8065-9037 https://orcid.org/0000-0002-7427-7059 https://orcid.org/0000-0002-5953-3843 https://orcid.org/0000-0001-8390-6032 https://orcid.org/0000-0003-3294-8990 https://orcid.org/0000-0003-4507-5412 https://orcid.org/0000-0001-5040-2938

\section{REFERENCES}

1. Furukawa T, Oohashi K, Watanabe Y, Yamao T, Katoh T, Yanagisawa A. [The usefulness of endoscopic resection of the adenoma of the papilla of Vater]. Gastroenterological Endoscopy 1999;43:284-295.

2. Binmoeller KF, Boaventura S, Ramsperger K, Soehendra N. Endoscopic snare excision of benign adenomas of the papilla of Vater. Gastrointest Endosc 1993;39:127-131.

3. Norton ID, Gostout CJ, Baron TH, Geller A, Petersen BT, Wiersema MJ. Safety and outcome of endoscopic snare excision of the major duodenal papilla. Gastrointest Endosc 2002;56:239-243.

4. Cheng CL, Sherman S, Fogel EL, et al. Endoscopic snare papillectomy for tumors of the duodenal papillae. Gastrointest Endosc 2004;60:757764 .

5. Bohnacker S, Seitz U, Nguyen D, et al. Endoscopic resection of benign tumors of the duodenal papilla without and with intraductal growth. Gastrointest Endosc 2005;62:551-560.

6. Catalano MF, Linder JD, Chak A, et al. Endoscopic management of adenoma of the major duodenal papilla. Gastrointest Endosc 2004;59:225232

7. Igarashi $\mathrm{Y}$, Okano N, Satou D, et al. Endoscopic snare excision for a major papilla tumor. Dig Endosc 2005;17:179-182.

8. Seifert E, Schulte F, Stolte M. Adenoma and carcinoma of the duodenum and papilla of Vater: a clinicopathologic study. Am J Gastroenterol 1992;87:37-42.

9. Stolte M, Pscherer C. Adenoma-carcinoma sequence in the papilla of Vater. Scand J Gastroenterol 1996;31:376-382.

10. Hirao M, Masuda K, Asanuma T, et al. Endoscopic resection of early gastric cancer and other tumors with local injection of hypertonic saline-epinephrine. Gastrointest Endosc 1988;34:264-269.

11. Kanda Y. Investigation of the freely available easy-to-use software 'EZR' for medical statistics. Bone Marrow Transplant 2013;48:452-458.

12. Han J, Kim MH. Endoscopic papillectomy for adenomas of the major duodenal papilla (with video). Gastrointest Endosc 2006;63:292-301.

13. Espinel J, Pinedo E, Ojeda V, Guerra Del Río M. Endoscopic ampullectomy: a technical review. Rev Esp Enferm Dig 2016;108:271-278.

14. Desilets DJ, Dy RM, Ku PM, et al. Endoscopic management of tumors of the major duodenal papilla: refined techniques to improve outcome and avoid complications. Gastrointest Endosc 2001;54:202-208.

15. Bassan M, Bourke M. Endoscopic ampullectomy: a practical guide. J Interv Gastroenterol 2012;2:23-30.

16. Hyun JJ, Lee TH, Park JS, et al. A prospective multicenter study of submucosal injection to improve endoscopic snare papillectomy for ampullary adenoma. Gastrointest Endosc 2017;85:746-755.

17. ASGE Standards of Practice Committee; Chathadi KV, Khashab MA, et al. The role of endoscopy in ampullary and duodenal adenomas. Gastrointest Endosc 2015;82:773-781.

18. Ito K, Fujita N, Noda Y. Endoscopic diagnosis and treatment of ampullary neoplasm (with video). Dig Endosc 2011;23:113-117.

19. Harewood GC, Pochron NL, Gostout CJ. Prospective, randomized, controlled trial of prophylactic pancreatic stent placement for endoscopic snare excision of the duodenal ampulla. Gastrointest Endosc 2005;62:367-370.

20. Dumonceau JM, Andriulli A, Deviere J, et al. European Society of Gastrointestinal Endoscopy (ESGE) guideline: prophylaxis of post-ERCP pancreatitis. Endoscopy 2010;42:503-515.

21. Irani S, Arai A, Ayub K, et al. Papillectomy for ampullary neoplasm: results of a single referral center over a 10-year period. Gastrointest Endosc 2009;70:923-932.

22. Aiura K, Imaeda H, Kitajima M, Kumai K. Balloon-catheter-assisted endoscopic snare papillectomy for benign tumors of the major duodenal papilla. Gastrointest Endosc 2003;57:743-747. 